ANL-HEP-CP-96-57

August, 1996

\title{
A lattice formulation of chiral gauge theories*
}

\author{
Geoffrey T. Bodwin ${ }^{\mathrm{a}}$ \\ a Argonne National Laboratory, \\ 9700 S. Cass Ave., Argonne, IL 60439, USA
}

We present a method for implementing gauge theories of chiral fermions on the lattice.

A more detailed account of many of the ideas presented here and a more complete set of references can be found in Ref. [1].

\section{THE LATTICE AS A UV REGULA- TOR}

The essential criterion for evaluating the suitability of a lattice formulation of a field theory is the extent to which the lattice fulfills the rôle of a UV regulator. A good UV regulator has the following properties. (1) The noninteracting theory yields the correct spectrum as the regulator is removed $(a \rightarrow 0)$. (2) The interacting theory yields the correct action as the regulator is removed, holding field momenta fixed (and neglecting irrelevant operators). (3) The regulated theory respects the "important" symmetries.

A theory that exhibits property (2) respects the symmetries of the unregulated (continuum) theory as the regulator is removed, holding field momenta fixed. However, if momenta of order the cutoff are important, these symmetries can be violated even once the regulator has been removed. The symmetry violations arise from UV-divergent amplitudes, and, hence, they correspond to shortdistance effective interactions (local renormalization counterterms).

There are many important symmetries. However, in discussing gauge theories, we focus on the gauge symmetry because it is a powerful tool to proscribe symmetry-violating counterterms. The gauge symmetry generally allows only those counterterms, such as coupling-constant renormalization, that are compatible with the symmetries of the continuum theory. Note that invariance un-

\footnotetext{
*Talk presented at the Lattice '96 Symposium, St. Louis, Missouri, June, 1996.
}

der infinitesimal gauge transformations is usually sufficient to rule out gauge-variant counterterms.

\section{A MODEL: CHIRAL QED}

As a concrete example of a lattice chiral gauge theory, let us examine four-dimensional QED with left-handed couplings to the gauge field. We take the standard plaquette action for the gauge field, and we begin with the simplest ("naive") discretization of the gauged fermion action:

$$
\begin{array}{r}
S_{N}=a^{4} \sum_{x, \mu} \bar{\psi}(x) \gamma_{\mu} P_{L} \frac{1}{2 a}\left[U_{\mu}(x) \psi\left(x+a_{\mu}\right)\right. \\
\left.-U_{\mu}^{\dagger}\left(x-a_{\mu}\right) \psi\left(x-a_{\mu}\right)\right],
\end{array}
$$

where $P_{R / L}=(1 / 2)\left(1 \pm \gamma_{5}\right)$, and, as usual,

$U_{\mu}(x) \equiv \exp \left[\operatorname{iag} A_{\mu}\left(x+a_{\mu} / 2\right)\right]$.

This action has the well-known doubling problem, which violates property (1): the fermion propagator

$i S_{F}^{N}=\left[(1 / a) \sum_{\mu} i \gamma_{\mu} \sin \left(p_{\mu} a\right)\right]^{-1}$

has poles at $p_{\mu}=0$ and $p_{\mu}=\pi / a$. The doublers can be eliminated in the usual way by introducing a Wilson mass term

$$
\begin{gathered}
S_{W I}=a^{4} \sum_{x, \mu} \bar{\psi}(x) \frac{1}{2 a}\left[2 \psi(x)-U_{\mu}(x) \psi\left(x+a_{\mu}\right)\right. \\
\left.-U_{\mu}^{\dagger}\left(x-a_{\mu}\right) \psi\left(x-a_{\mu}\right)\right] .
\end{gathered}
$$

Now the propagator is

$i S_{F}^{W}=(1 / a) \sum_{\mu}\left[i \gamma_{\mu} \sin \left(p_{\mu} a\right)+M(p)\right]^{-1}$,

where $M(p)=(1 / a) \sum_{\mu}\left[1-\cos \left(p_{\mu} a\right)\right]$ is the Wilson mass. There is a pole only at $p_{\mu}=0$, and the lattice action satisfies properties 1 and 2 . 
However, the action does not satisfy property (3). The Wilson mass term violates the chiral symmetry, since it connects $P_{L} \psi$ to $\bar{\psi} P_{R}$. Consequently, the action is not invariant under an infinitesimal gauge transformation

$U_{\mu}(x) \rightarrow U_{\mu}(x)+i \Lambda(x) U_{\mu}(x)-i U_{\mu}(x) \Lambda\left(x+a_{\mu}\right)$,

$\psi(x) \rightarrow\left[1+i P_{L} \Lambda(x)\right] \psi(x)$,

which involves only the left-handed fermion field. The gauge variation of the action is nonzero because the Wilson mass operator commutes, rather than anticommutes, with $\gamma_{5}$.

The gauge variation gives a $\Lambda$-fermion vertex, which, in momentum space, is

$\mathcal{M}=i\left(P_{R}-1\right) M(p)-i\left(P_{L}-1\right) M(p+l)$,

where $p$ is the fermion momentum, and $l$ is the gauge-field momentum. Now, $M(p)$ vanishes as $a p^{2}$ in the limit $a \rightarrow 0$ with $p$ fixed. Therefore, gauge variations can survive in the continuum limit only if $p$ or $l$ of order $1 / a$ are important, i.e., only in UV-divergent expressions.

\section{MODIFICATION OF THE FERMION DETERMINANT}

We can try to restore the gauge symmetry by modifying fermion amplitudes. Since the difficulties arise from the fact that $\gamma_{5}$ commutes with the Wilson term, let us adopt the strategy of treating $\gamma_{5}$ as if it anticommuted with the Wilson term. Of course, we make an error in doing this. However, the error is proportional to Wilson terms, which vanish as $a \rightarrow 0$, unless $p \sim 1 / a$. Therefore, the procedure amounts to adding local interactions (counterterms) to the theory, i.e., to modifying the UV regulator.

Consider a typical fermion-loop amplitude:

$\operatorname{Tr} \ldots\left(\not p_{1}+M\right)^{-1} \Gamma_{\mu} P_{L}\left(p_{2}+M\right)^{-1} \Gamma_{\nu} P_{L} \ldots$,

where the $\Gamma$ 's are gauge-field-fermion vertices and we are using a schematic notation for the fermion propagators. For even-parity terms, (those containing an even number of $\gamma_{5}$ 's), we treat $\gamma_{5}$ as if it commuted with all propagators and vertices, including Wilson terms, and use the identity $\gamma_{5}^{2}=1$ to eliminate $\gamma_{5}$ 's. The even-parity part of the amplitude is then

$$
(1 / 2) \operatorname{Tr} \ldots\left(\not p_{1}+M\right)^{-1} \Gamma_{\mu}\left(\not p_{2}+M\right)^{-1} \Gamma_{\nu} \ldots
$$

This is $1 / 2$ the amplitude for a Dirac particle with vector-like coupling to the gauge field. Now, the vector-like amplitude is gauge invariant, provided that we gauge the Wilson term. (In this case we must transform $\psi$, not just $P_{L} \psi$.) Since $\operatorname{det} \mathcal{D}=\exp (\operatorname{Tr} \ln \mathcal{D})$, where $\operatorname{Tr} \ln \mathcal{D}$ is the amplitude, $1 / 2$ the amplitude corresponds to the square root of the fermion determinant. It is easy to see that the even-parity part of the amplitude yields the magnitude of the determinant. Therefore, we can render the magnitude of the determinant exactly gauge invariant by replacing it with the square root of the determinant for a Wilson-Dirac particle with vector-like coupling to the gauge field. (There is a similar modification for interacting fermion propagators 酒.)

In the case of the odd-parity terms, this trick doesn't work. There is always one $\gamma_{5}$ that can't be eliminated. However, the Dirac trace of an odd number of $\gamma_{5}$ 's is proportional to $\epsilon_{\mu \nu \rho \sigma}$. Therefore, as $a \rightarrow 0$ with gauge-field momenta fixed, the gauge variation is proportional to operators with dimension less than five containing $\epsilon_{\mu \nu \rho \sigma}$. These are precisely the operators that correspond to the ABJ anomaly. Thus, anomaly species cancellation guarantees the gauge symmetry for the odd-parity loop amplitudes - as long as we consider only gauge-field configurations with momenta much less than $\pi / a$.

\section{LARGE GAUGE-FIELD MOMENTA}

In odd-parity contributions, gauge-field momenta $l \sim 1 / a$ flowing through a fermion line yield nonvanishing gauge variations, since $M(p+$ $l$ ) is not negligible. Gauge-field momenta $l \sim 1 / a$ are important in UV-divergent loops involving gauge fields. Such loops generate all local interactions that are consistent with the symmetries of the lattice theory. Many of the interactions are gauge variant and must be eliminated-possibly by tuning of the corresponding counterterms.

Let us now discuss a method to avoid explicit tuning of counterterms. The essential idea is to 
introduce separate lattice spacings (cutoffs) $a_{g}$ for the gauge field and $a_{f}$ for the fermion field. Then $a_{f}$ is taken to zero before $a_{g}$. The goal of such a procedure is to suppress amplitudes in which gauge-field momenta are greater in magnitude than $\pi / a_{g}$. Then Wilson terms in propagator numerators and vertices can be neglected, except in divergent fermion loops, which we have already dealt with by modifying the fermion determinant and invoking anomaly cancellation.

This idea is implemented as follows. One treats links on the $a_{g}$ lattice as the dynamical variables: the gauge-field action depends only on these links. The fermion-field action-including the interactions with the gauge field - resides on the $a_{f}$ lattice. One obtains the links on the $a_{f}$ lattice by interpolating the gauge fields on the $a_{g}$ lattice.

A good interpolation must have certain properties: (1) gauge covariance; (2) continuity on the $a_{f}$ lattice, except at boundaries of $a_{g}$ plaquettes, (3) locality. Property (1) means that a gauge transformation on the $a_{g}$ lattice maps into a gauge transformation on the $a_{f}$ lattice. Property (2) guarantees that the Fourier transform of the gauge field is suppressed as $1 /\left(a_{g} l\right)$ for gauge-field momenta $l \gg 1 / a_{g}$. Property (3) insures that one recovers the continuum theory for $l a_{g} \ll 1$. Under these assumptions, one can prove, to all orders in perturbation theory, that, as $a_{f} \rightarrow 0$ with $a_{g}$ fixed, gauge variations vanish as $a_{f}$, and deviations from the target theory vanish as $a_{f}^{2}$ (Ref. [1,2]). This result also holds in the presence of individual nonperturbative gaugefield configurations [1].

We assume that the lattice origins coincide and that $a_{g} / a_{f}$ is an integer. Along $a_{g}$-lattice links $U_{g}$, we take the gauge-field on the $a_{f}$ lattice, $A_{f}$, to be

$A_{f \mu}=\left(1 / i a_{g} g\right) \log U_{g \mu}$,

which is consistent with gauge covariance. Away from the $a_{g}$ links, one obtains $A_{f \mu}$ by interpolation, using (10) as a boundary condition. For Abelian gauge groups, a simple linear interpolation is consistent with the properties of a good regulator. (For non-Abelian groups the interpolation is more complicated [2]). The links on the $a_{f}$ lattice are then obtained from $A_{f}$.

\section{A NONPERTURBATIVE PROBLEM}

Shamir has pointed out a potential difficulty with interpolation of the gauge field [3]. A gauge transformation on the $a_{g}$ lattice $U_{\mu}(x) \rightarrow$ $V^{-1}\left(x+a_{g \mu}\right) U_{\mu}(x) V(x)$ can take $U$ through -1 when either the transformation is large or $\mathrm{U}$ is near -1 . Then, $g A_{f \mu}$ (Eq. 10) jumps from $\pi / a$ to $-\pi / a$. The jump corresponds to a change in the winding number. Hence, the corresponding gauge transformation on the interpolated field is singular. Therefore, we are faced with two alternatives: require gauge covariance and accept that the interpolated field may be singular, or require smoothness of the interpolated field and accept that gauge covariance is violated for some gauge transformations.

Let us adopt the second alternative. (In the Abelian case, we simply take the linear interpolation of (10).) Then we still have invariance under infinitesimal gauge transformations, except at $g A_{\mu}=\pi / a$. Recall that we require only invariance under infinitesimal transformations to prohibit undesirable counterterms. The issue then is whether the point $g A_{\mu}=\pi / a$ important in the path integral.

To address this question, let us consider, for example, the situation in the gauge $\nabla_{\mu} A_{\mu}=0$. In this gauge $\nabla^{2} A_{\mu}=J_{\mu}$, so $A_{\mu} \sim 1 / a$ can arise only from currents (momenta) $\sim 1 / a$. The most divergent amplitude is the vacuum polarization. By dimensional analysis the vacuum polarization $\Pi_{\mu \nu}$ goes as $\delta_{\mu \nu}\left(1 / a_{g}^{2}\right)$. However, invariance under infinitesimal gauge transformations reduces this to $\Pi_{\mu \nu} \sim\left(k^{2} \delta_{\mu \nu}-k_{\mu} k_{\nu}\right) \log \left(1 / a_{g}\right)$. This result can be verified by explicit calculation in the Abelian theory with the linear interpolation. Therefore, $A_{\mu}$ grows only as $\log (1 / a)$. We conclude that the point at $g A_{\mu}=\pi / a$ doesn't contribute significantly to the path integral as $a \rightarrow 0$.

\section{REFERENCES}

1. G.T. Bodwin, hep-lat/9510002, to be published in Phys. Rev. D.

2. P. Hernández and R. Sundrum, Nucl. Phys. B455 (1995) 287 (hep-ph/9506331).

3. Y. Shamir, hep-lat/9509023. 